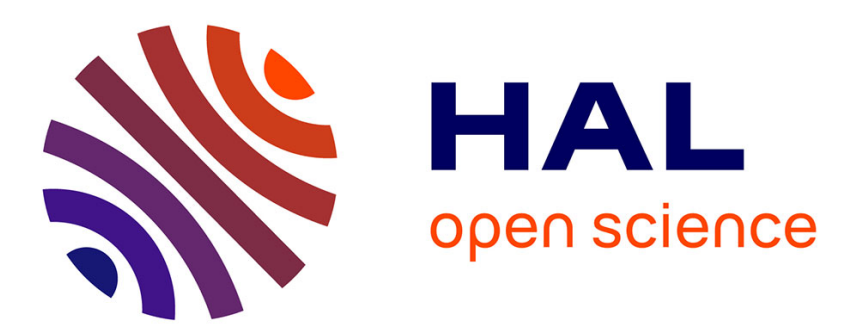

\title{
Digital Sketching for distant collaborative diagnosis in neurosurgery: an experimentation
}

\author{
Stéphane Safin, Felix Scholtes, Pierre Bonnet, Pierre Leclercq
}

\section{To cite this version:}

Stéphane Safin, Felix Scholtes, Pierre Bonnet, Pierre Leclercq. Digital Sketching for distant collaborative diagnosis in neurosurgery: an experimentation. IEA 2018: 20th Congress of the International Ergonomics Association, Aug 2018, Florence, Italy. 10.1007/978-3-319-96077-7_58 . hal-02085957

\section{HAL Id: hal-02085957 \\ https://hal.science/hal-02085957}

Submitted on 1 Apr 2019

HAL is a multi-disciplinary open access archive for the deposit and dissemination of scientific research documents, whether they are published or not. The documents may come from teaching and research institutions in France or abroad, or from public or private research centers.
L'archive ouverte pluridisciplinaire HAL, est destinée au dépôt et à la diffusion de documents scientifiques de niveau recherche, publiés ou non, émanant des établissements d'enseignement et de recherche français ou étrangers, des laboratoires publics ou privés. 


\title{
Digital Sketching for distant collaborative diagnosis in neurosurgery: an experimentation
}

\author{
Stéphane Safin ${ }^{[1]}$ Felix Scholtes ${ }^{[2]}$, Pierre Bonnet ${ }^{[2]}$ and Pierre Leclercq ${ }^{[3]}$ \\ ${ }^{1}$ I3-SES, CNRS, Télécom ParisTech, 75013, Paris, France \\ ${ }^{2}$ CHU, University of Liège, Belgium \\ ${ }^{3}$ LUCID, Faculty of Applied Sciences, University of Liège, Belgium \\ stephane.safin@telecom-paristech.fr
}

\begin{abstract}
This study relates an experiment about multimodal distant collaboration in neurosurgery. We observed a two-hours distant meeting between neurosurgeons, supported by an original platform allowing to convey remotely speech and gesture (videoconferencing) but also images and hand drawn sketches. We use a bottom-up approach, analyzing and understanding the spontaneous activity set up by the professionals. We show that conversations about diagnosis and operational strategies are intimately linked, that different communication modalities are preferred to support different communication topics, and that digital Sketch is a flexible tool in remote collaboration in medicine.
\end{abstract}

Keywords: Remote collaboration, Digital Sketch, Neurosurgery.

\section{Introduction}

Nowdays, in a wide range of activity sectors, collaboration between actors has intensified. In particular, in the medical field, the over-specialization of professionals, the merging of hospital institutions, and the need to cross skills to refine diagnoses and define optimal therapeutic strategies, necessarily raise the issue of effective remote collaboration. In this context, and with a solid experience of distant collaboration in the field of architecture, we are specifically interested in understanding and supporting remote synchronous collaboration, particularly in the field of neurosurgery.

In this study, we address the following questions: how can digital sketch effectively support distant collaboration in medical domain? How do expert practitioners rely on several communication modalities (verbal, gestural, visual) to build, remotely, a joint understanding and a common approach to medical challenges? What is the relation between communication modalities and contents?

The study consists in the observation of a remote collaborative session between two neurosurgeons based in Liège, and one in Montreal, all three expert practitioners. The discussion is free, addresses three clinical cases and is supported by SketSha, a digital freehand sketching software. Originally developed for architecture, combined with a 
conventional video conferencing system, it constitutes a multimodal communication device conveying speech, gestures, drawing and professional content (medical imaging). Neurosurgery, as a discipline that relies heavily on medical imaging, is an appropriate field for testing new forms of graphical interaction.

The objective of this two-hour meeting was multiple: to experiment the device in the medical field, to mobilize it in a real medical collaboration activity and to document this activity, to identify the potentialities of the system in the field and to imagine uses for longer term.

\section{Collaborative practices in medicine}

Diagnostic and therapeutic medical decisions are based on a bundle of diverse information most often analyzed collegially, in real time, only if the stakeholders are together. A legal framework now imposes these collegial practices in certain situations such as the management of oncological pathologies (Multidisciplinary Oncology Consultation). Thus, recommendations for good medical practice go through a Peer-review system that sometimes takes on an international dimension. These collegial analyzes therefore imply that physicians (most of whom are specialists with high added value) would have either to travel over sometimes enormous distances, which implies an important cost, or to make these meeting in degraded conditions, by phone for example. In view of the difficulties of time and resources, these collegial meetings are limited in number and / or participants, to the potential detriment of the medical quality of patients' care.

In addition, the hospital landscape has evolved. The institutions have merged into hospital groups located in several geographically distant locations. Each location often has a specific, oriented activity.

Medical practice, especially that of adequate diagnosis, leading to the possibility to devise treatment strategies, relies on medical data of various kinds: biological data, medical history and medical imaging. Medical imaging has evolved considerably. This includes the way high resolution tomographic images are delivered to the practitioner. Today, diagnosis is based on the viewing of series of images, merged images and reconstructions. Analyzing a complex imaging case may require navigating through hundreds of images, using multi-planar reconstructions, and measurement tools in some cases. More and more often medical experts analyze these images collegially. The written analysis from the medical imaging specialist is not sufficient, the decisions being often the result of a collective intelligence. In addition, the images are often manipulated and annotated to integrate therapeutic decisions. In the field of surgery, this makes it possible, for example, to delineate the area of tumor resection or sketch the approach for vascular lesions.

This evolving landscape and potential requirements of new practices require the use of adapted exchange tools. Videoconferences which allow synchronous collegial work between geographically distant teams partially respond to these requirements, but lack flexibility and functionality: the need for dedicated adapted premises, the impossibility 
of producing graphic annotations collaboratively and remotely. In order to fulfil existing requirements and potentially drive development of multi-modal long-distance collaboration between experts, It is therefore crucial to have effective remote collaboration tools, and in particular synchronous collaboration tools, to equip these growing collaborative practices and support the transitions in the hospital field.

\section{Digital Sketching and SketSha software}

Sketching has been recognized as a powerful tool for expressing ideas in the design domain: by its ambiguous character, it supports creativity, and by its easiness of expression, it allows problem decomposition (Goldschmidt, 1991). Therefore, several software and environments tend to combine these intrinsic properties of hand-drawn sketch with digital support. This is known as the paradigm of Digital Sketch.

In order to support remote synchronous collaboration, the LUCID-ULg lab has developed a software called SketSha (for sketch sharing), as a shared drawing environment allowing several users to be connected to the same virtual drawing space. Various functionalities, such as a panel of colored pens (and an eraser) and a navigation tool (to zoom, translate, rotate), are available through intuitive graphical widgets and a digital pen. Some layout facilities have also been included in the prototype, such as the possibility of drawing and managing different sheets of virtual paper, of deleting or duplicating them, and of managing their transparency. The software is installed on a graphic tablet and completed with a video-conferencing system, for remote collaboration (see figure 1)
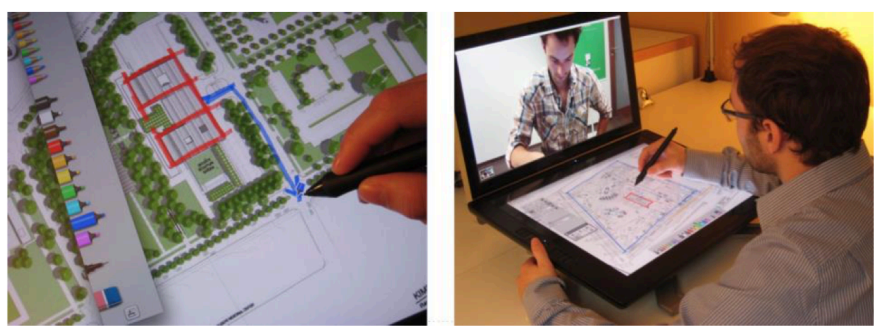

Fig. 1. SketSha environment (used in design domain)

The system has been intensively used in architectural professional and educational settings (Dondero \& Shirkhodaei, 2014; Safin et al., 2011). These uses have proven its efficiency to support distant flexible and rich collaboration in design domain (Safin 2011, Safin et al. 2012), but have also opened the doors to the exploration of digital sketch as a support to remote collaboration in other domains, such as medicine. This is the aim of this study. 


\section{Research issue}

In this study, we experiment the use of SketSha environment in a medical context. We use a bottom-up approach: we propose the system, support the technical setting and let the users build their own collaborative activity around the system. The research questions are twofold

- On a pragmatic/applied point of view, we aim to identify the potentialities of digital sketch for collaboration in the medical domain and to define requirements for effective sketch-based remote medical collaboration, by documenting spontaneous collaborative activities in the field.

- On a fundamental point of view, we aim to analyze an original collaborative practice (remote sketch-based collaboration in medicine) to address the following questions

- How do expert practitioners rely on several communication modalities (verbal, gestural, visual) to create a common diagnosis remotely?

- What is the relation between communication modalities and contents?

- How is the remote collaboration activity spontaneously structured?

For this purpose, following Rabardel (1995) notion of instrument, we provided users with an artifact (the SketSha environment, initially developed for architecture and design, and probably poorly fitted to medical practices) and document the instrumental genesis processes, i.e. the way this artifact is modified, diverted, appropriated, and the way uses and activities are re-built around this artifact.

\section{$5 \quad$ Setting}

The study consists in the observation of a remote collaborative session between two neurosurgeons based in Liège, and one in Montreal, all three confirmed practitioners (Figure 2). The discussion is free and addresses three patients' cases. The goal for practitioners are to confirm the diagnosis, discuss operative indications and strategies and share best practices.
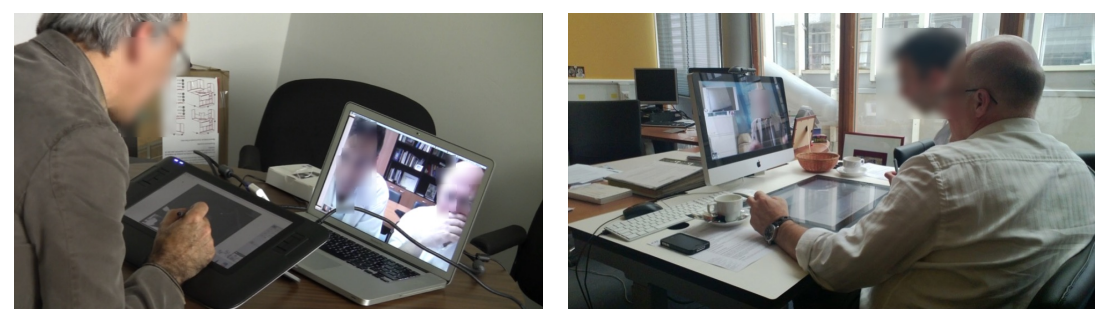

Fig. 2. Remote collaboration between Montreal (left) and Liège (right) 
During the meeting, which lasted about two hours, the observed professionals discuss cases, manipulate videos (collection of images from medical imaging devices ${ }^{1}$., extract still images on the system, draw on these images, point to elements with the pen (the cursor is reproduced in the remote environment) and make gestures through videoconferencing (fig.3). The three patient cases discussed at this meeting allowed practitioners to discuss about care strategies (embolization, surgery, etc.) and about best practices in surgery (position of the surgeon for the operation, type of craniotomy, instruments used, etc.)

Beyond case discussions, we observe reflexive moments where the participants explain of the use of the device, they express comments about it, and where they consider intensive use of the device for their professional practice.
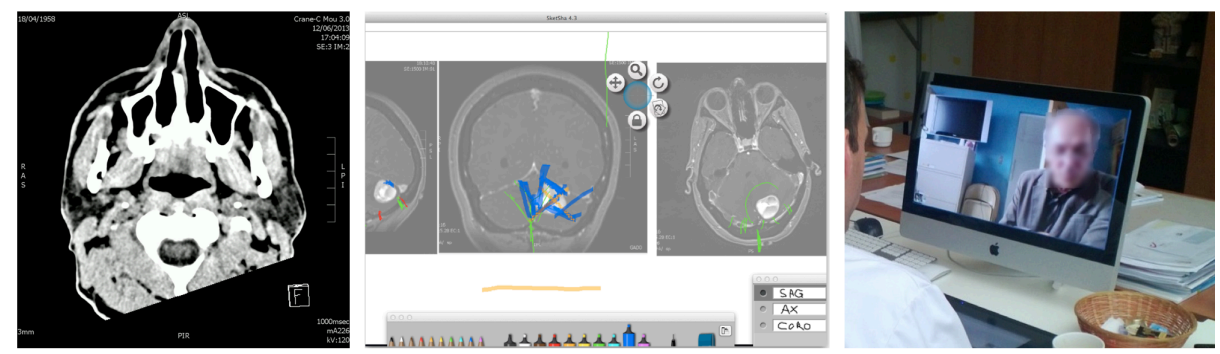

Fig. 3. Information channels: videos composed of medical imagery (left), digital sketches on imagery (center), videoconference (right)

\section{Methodology}

The video of the collaborative activity was analyzed in depth, based on ad-hoc coding grids, covering the following aspects

At first, we identify the type of activities which take place, with the following categories: Case discussions (the core activity); Organization of the collaboration (explicitation of collaboration strategies); Preparation of discussion material (choice of videos, selection, import of images into SketSha); Logistical feedbacks (explanation on the device, spontaneous comments from users on the device, technical problems); Projection in the future (moments of activity recomposition, feedback on the activity progress and projection in probable future activity); and Informal discussions (humor, etc.).

Secondly, we note multimodal actions: Manipulation of videos; pointing gestures (pen passing over the image); annotations (drawings); figurative gestures, i.e. which participate in an expression of a content, and not only in the punctuation of the communication. It should be noted that, for technical reasons, these actions have only been identified for the Montreal partner.

\footnotetext{
${ }^{1}$ Each image is a cross-section of the considered anatomic element, and the viewing of the video can simulate the navigation on the set of images, which is a usual way for neurosurgeons to apprehend the three-dimensional aspects of a case in a large anatomic imaging set.
} 
Third, for the sequences bearing only case discussions (the majority of the activity), we operate a very simple classification of topics of discussion according to two crossed dimensions (4 categories) (1) Epistemic vs. pragmatic (Rabardel \& Folcher, 2004): on the one hand the elaboration or the evocation of knowledge (diagnosis, anatomy, etc.), on the other the discussions on action modalities (surgical procedures, materials to use, etc.) and (2) Particular vs. General depending on one refers to the current case or to general questions. By crossing these two dimensions we obtain the following table

Table 1. discussion topics.

\begin{tabular}{|l|l|l|}
\hline & Epistemic & Pragmatic \\
\hline General & Anatomy & General requirements on surgery processes \\
\hline Particular & Current case diagnosis & $\begin{array}{l}\text { Actions to be planned for the current case. } \\
\text { Preparation of surgery }\end{array}$ \\
\hline
\end{tabular}

All these actions have been coded on activity video, for each second. By crossing of these different variables, we tackle questions related to the strategies for articulating different contents in medical collaboration, to the contribution of the different communication modalities in distant communication, to the evolution of communication and system appropriation, to the issues and opportunities related to the mobilization of the digital sketch for remote medical collaboration. When appropriate, we calculate Relative Deviations (RDs) ${ }^{2}$ that measure local associations between specific modalities of each of the two variables

\section{$7 \quad$ Results}

\subsection{Global activity structure and description}

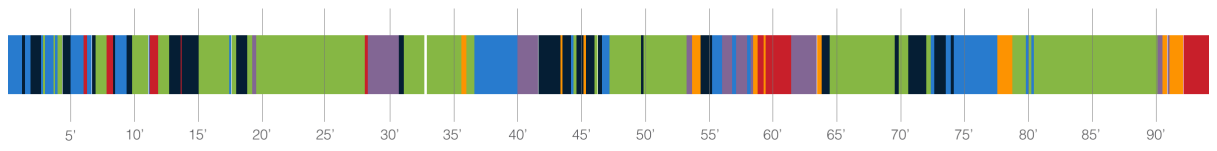

Fig. 4. Timeline of actions: Discussion (green), organization (red), preparation (black), logistics (blue), projection (purple) and informal (orange)

Figure 4 shows the sequence of the types of activities. Globally, task-oriented activities represent $46 \%$ of the time, process-oriented activities (operating synchronization: preparation and organization) represent $23 \%$ of the time, communication management activities and feedbacks represent $26 \%$ (logistic and projection) and social-emotional processes represent $5 \%$ of the discussion time. By comparing the proportion of different

2 RDs measure the association between two nominal variables. They are calculated on the basis of a comparison between observed and expected frequencies (i.e. those that would have been obtained if there was no association between the two variables). There is attraction when the $\mathrm{RD}$ is positive, and repulsion - when it is negative. We considered a threshold of 0,5 (in absolute value) to consider it as a strong association. 
activities in the two halves of the session, we obtain the following observations (Table 2 ). The proportion of time devoted to the core of the activity remains stable, but the logistics and preparation activities are drastically reduced in the second half of the activity, indicating a habituation to the system. Informal activities (humor), on the other hand, increase as does the organization (which is mainly a long discussion on the actions to be put in place in the short and medium term, closing the meeting). So there is a fairly clear learning curve of the use of the system.

Table 2. types of activities and evolutions.

\begin{tabular}{|l|l|l|l|}
\hline & First Half & & Second half \\
\hline Discussion & 0,47 & $=$ & 0,45 \\
\hline Organization & 0,03 & + & 0,14 \\
\hline Preparation & 0,20 & - & 0,10 \\
\hline Logistic & 0,20 & - & 0,12 \\
\hline Projection & 0,09 & $=$ & 0,11 \\
\hline Informal & 0,02 & + & 0,09 \\
\hline TOTAL & 1 & & 1 \\
\hline
\end{tabular}

The activity can be divided into five phases (Figure 5):

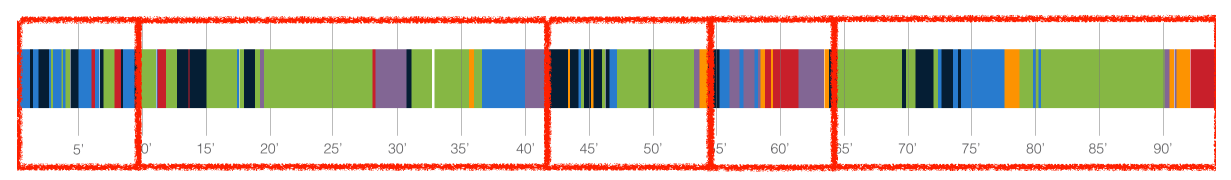

Fig. 5. Five phases of the activity

A first phase, at the beginning of the meeting, is logically dominated by logistical issues and meeting preparation. This phase consists in the training of the users, as well as the preparation of the first images. This training session lasts about 10 minutes, which seems sufficient to apprehend the system.

There follows a first round of discussion on the first case: subarachnoid hemorrhage with large aneurysm. This first cycle starts with preparation, continues with the discussion of the case (on the therapeutic strategy, the operative strategy, the operating modes, and the instruments and tools to be used for this type of case), interrupted by a moment when the users spontaneously propose uses of the communication device and ways of using it, especially for teaching. The cycle closes with an evaluation of the system by users and suggestions for improvement (logistics), followed by proposals for uses (projection).

The second round of discussion is about another case of aneurysm (reflection on the strategy to use, surgical or endovascular, treatment delays, recovery between two surgeries) contains a preparation step followed by a long discussion. We also see in this second cycle "informal" moments, humor and off-topic discussions, reflecting a relaxed atmosphere. This social element is a crucial issue of remote collaboration, and seems appropriately supported by the system. 
In the next stage, there is an explicit feedback on the collaborative experimentation, with a mixture of projections into the future, logistical feedbacks or clarification, and organization of the continuation of the session.

The third round of discussion, preceded by a very short preparation, is essentially productive. It focuses on an intra-axial tumor with edema, and professionals discuss the therapeutic strategy and the operative strategy. It is interrupted by a logistic cut to select other images. There are only a few moments of projection in the possible future activity and informal communications continue to punctuate the sequence.

\subsection{Topics}

In the first round of case discussions, the collaborators make a global movement from the particular / heuristic to the general / pragmatic through episodes of particular / pragmatic. In other words, they rely on information about the current patient's case (part / heur), then discuss the modes of intervention to be mobilized for this case (part / prag) to finish with more general considerations on operations of this type (gen / prag).

The second cycle, shorter, also includes the same movement, but the points of view discussed are a little more mixed up with each other. In the third round of discussion, most of the time is spent on the diagnosis on the specific case (part / heur) before concluding very briefly by discussions of type part / prag then gen / heur. Finally, the fourth cycle is essentially pragmatic, from the particular to the general. The general repartition of discussion topics is displayed in table 3 .

Table 3. Proportion of topics

\begin{tabular}{|l|c|c|}
\hline & Epistemic & Pragmatic \\
\hline General & 0,04 & 0,38 \\
\hline Particular & 0,30 & 0,28 \\
\hline
\end{tabular}

\subsection{Actions and communication modalities}

The work of the neurosurgeons is essentially verbal: $78 \%$ of the working time is done without other actions. The handling of videos is $9 \%$ of working time, drawings $7 \%$, gestures $4 \%$ and pointing $2 \%$. The videos are composed of a succession of cross-section. The manipulation of a set of images is a usual way for neurosurgeons to understand the three-dimensional aspects of the cases. The drawing proportion concerns only the moments where sketches are traced and do not include moments where sketches are viewed. The gestures refer to figurative gestures (to mimic a surgical gesture for example). Finally, these actions only concern the Montréal partner. Although being quite relative, these numbers clearly show a predominance of verbal interactions punctuated by complementary actions of different types. 
These actions differ depending on the type of discussions. The intensity of the link is strong (link rate, $\mathrm{V} 2=0.16$ ). The relative deviations are indicated in colors $(0.25$ threshold) in red for repulsions and in green for attractions in table 4 .

Table 4. links between actions and topics

\begin{tabular}{|l|l|l|l|l|}
\hline & Drawings & Gestures & Pointing & Video \\
\hline General/epistemic & 0,01 & 0,04 & 0,36 & 0,10 \\
\hline General/pragmatic & 0,32 & 0,85 & 0,25 & 0,09 \\
\hline Particular/epistémic & 0,43 & 0,00 & 0,24 & 0,59 \\
\hline Particular/pragmatic & 0,23 & 0,11 & 0,15 & 0,23 \\
\hline Total & 1 & 1 & 1 & 1 \\
\hline
\end{tabular}

Drawing thus has a polyvalent function, and serves to support all types of speech, with the exception of general considerations on anatomy. Gestures are clearly preferentially mobilized to support the discourses concerning general considerations on the neurosurgical practice. It is essentially used to figure and mimic operative gestures. The pointing gestures are quite infrequent, and support essentially general topics, to support discourses by highlighting some elements of the image. It seems that sketching and pointing are complementary: when a user needs to highlight information related to the current case, he draws, leaving a trace, but when he uses current image to support general considerations, he points with the stylus, leaving no trace on the drawing area. Finally, videos are mostly used to talk about the current case and support very few general pragmatic considerations.

On the use of drawing, users make spontaneous comments quite positive: "For the past few months, videoconferences have been used, a monthly meeting with our colleagues [...] And that's pretty much what we do, we present each other patients and then we discuss. And I see the difference. I am very happy to use this system today because I see the difference and the potential that we can have in drawing at a distance, because we have one more element that I think could be very important, especially if we had an image database»

In addition, the users look a lot through videoconferencing, unlike what has already been observed in collaborative activities in the field of architecture (Mayeur et al., 2010). They explain this because the images used and manipulated "do not represent exactly what one is talking about" they are a support for the discussion, but the information is primarily verbal.

\section{Conclusions}

The SketSha system, originally designed for architecture, has a number of domain-specific features: the system is designed to import a small number of large images (plans) on which professionals must work for relatively long durations. However, in the field of Neurosurgery, we see that it is necessary to manipulate many images (scanner crosssections) and work on it for shorter periods. This requires, for an adaptation to the field, 
important modifications of the device, such as a specific management of medical images. At this stage, and unsurprisingly, the relative importance of logistics and preparation activities is detrimental to the smooth use of the communication device. On the other hand, the relatively short appropriation time of the device and the spontaneous construction of new communication practices based on the sketch testify to a promising nature for this type of device. In addition, the social-affective processes of collaboration can be supported with this type of multimodal environment.

From a more fundamental point of view, this study is a unique experiment providing some insights on the collective practice of medical diagnosis and treatment strategies elaboration, when it is held by remote practitioners, and aims to understand how professionals spontaneously construct uses of a multimodal device and structure their collaborative activity. We identify different movements in the discussion of a case in neurosurgery. Although each case is approached differently, we see movements starting with the diagnosis of the current case, supported by the images, continuing towards the determination of the operating strategies for this case, supported by the freehand drawing, and ending with the sharing of general experiences on the modes of operation, supported by the gestures. Our study thus shows the potential of the sketch for distant communication in neurosurgery, supporting a versatile role in communication, complementary to pointing gestures. In addition, the sketch appears the privileged means, in combination with the dynamic medical images, to work on the particular / pragmatic dimensions, i.e. on the definition of strategies and gestures to carry out during surgery. This is precisely the general objective pursued by meetings of specialists to prepare surgical procedures.

Finally, the users propose also to use the device for distance training in anatomy. It would involve setting up discussions with less experienced professionals around an images database. As we have seen in this study, pragmatic reflections are largely supported by the system: the gesture and the drawing allow to support long discussions useful for the learning of the profession of neurosurgeon.

Obviously, the study has several limitations: only one session was observed, with few training from the participants. Results may be different with "expert" use of the system, with more drawing and pointing, less logistical issues, etc. We need to engage in a more longitudinal study of the system use.

\section{$9 \quad$ References}

1. Dondero, M.G. \& Shirkhodaei, S. (2014). Appropriation et gestion d'une activité collaborative distante. Le cas d'un atelier pédagogique de la conception architecturale. Proceedings of COMMON 2014, Liège.

2. Folcher, V., \& Rabardel, P. (2004). Hommes, artefacts, activités : perspective instrumentale. In P. Falzon (Ed.), Ergonomie (pp. 251-268). Paris: PUF.

3. Goldschmidt, G. (1991). The dialectics of sketching. Creativity research journal, 4(2), 123143. 
4. Mayeur, A., Ben Rajeb, S., Darses, F., Lecourtois, C., Caillou, S., Guéna, F., Honigman, A., Leclercq, P. \& Safin, S. (2010) Concevoir à plusieurs et à distance en architecture : vers de nouvelles pratiques professionnelles ? Présentation au séminaire Globalisation et Territorialisation : questions de travail, Université Paris 1 Sorbonne, 7-9 juin.

5. Rabardel, P. (1995). Les Hommes et les Technologies. Approche Cognitive des Instruments Contemporains. Paris: Armand Colin.

6. Safin; S. (2011). Processus d'externalisation graphique dans les activités cognitives complexes : le cas de l'esquisse numérique en conception architecturale individuelle et collective. PhD Thesis, University of Liège

7. Safin, S., Kubicki, S., Bignon, J-C. \& Leclercq P. (2011). Digital Collaborative Studio : 4 years of practice. Poster presented at the CAAD Futures 2011 Conference, Liège, Belgium : July.

8. Safin, S., Juchmes, R. \& Leclercq, P. (2012). Use of graphical modality in a collaborative design distant setting. Proceedings of COOP 2012. Marseille, France, June. 